Journal of Mobile Communication 4 (2): 43-46, 2010

ISSN: 1990-794X

(C) Medwell Journals, 2010

\title{
Interference Suppression in Satellite Personal Communication Networks Using Adaptive Antenna Arrays and Filtering Technique
}

\author{
Sunday E. Iwasokun and Michael O. Kolawole \\ Department of Electrical and Electronics Engineering, \\ School of Engineering and Engineering Technology, \\ Federal University of Technology, Akure, Nigeria
}

\begin{abstract}
The study addresses the problem of interference as related to Satellite Personal Communication Networks (S-PCNs). Basic Low Earth Orbit (LEO) constellation is considered. The study uses combined adaptive antenna arrays and adaptive filtering technique. This hybrid linear adaptive technique provides improved performance eliminating interference, particularly uncorrelated signals residing in the antenna sidelobes.
\end{abstract}

Key words: S-PCN, LEO, adaptive array antennas, adaptive filtering, interference elimination, Nigeria

\section{INTRODUCTION}

A Satellite Personal CommunicationNetwork(S-PCN) provides universal accessibility to a wide range of services and across transnational boundaries. It faces multitude of challenges particularly those related to user terminals, the space segment, regulatory challenges (Ghedia, 1999) as well as technical issues including link diversity, traffic allocation (Septiawan, 2004) Quality of desired Service (QoS) and the associated Grade of Service (GoS) (Conte, 2005) etc.

The interference encroachment into the signals received by systems terminals impacts on the QoS and GoS as well and importantly the network congruous scope to deal with the motion of the satellite as well as that of the mobile user (Kolawole, 2009). Most mobile stations transceivers signals are ideally circularly polarized and isotropic in nature with a single low gain antenna element.

However, these single element units are particularly more susceptible to noise interference because they receive signals from all directions.

Adaptive antenna array mounted on the mobile unit is an approach suitable to combating interference problem as communication satellite traverses the sky which this study attempts to investigate. Of particular concern is the interference on voice channels causing crosstalk e.g., where the subscriber hears interference in the background. Figure 1

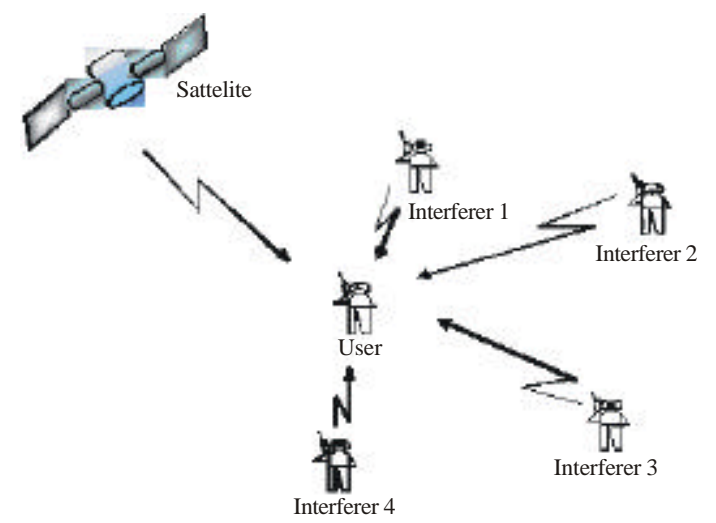

Fig. 1: S-PCN mobile user affected by interfernece

shows a S-PCN user affected by random, multidimensional interferences.

Array geometry: An adaptive array antenna unit is designed as in Fig. 2, bringing all the signals received by the various elements from a particular source into phase for further processing as well as achieving some desired performance, such as maximizing the received Signal-toNoise Ratio (SNR). Drawing from Skolnik (1981) and Kolawole (2003), we analyse the antenna array assuming that there is a direct path for the signal from the satellite and interfering signal is uncorrelated with the desired signal, all the array elements are placed closely enough such that there is no significant amplitude variation due to the difference in propagation path length for any two

Corresponding Author: Sunday E. Iwasokun, Department of Electrical and Electronics Engineering,

School of Engineering and Engineering Technology, Federal University of Technology, Akure, Nigeria 


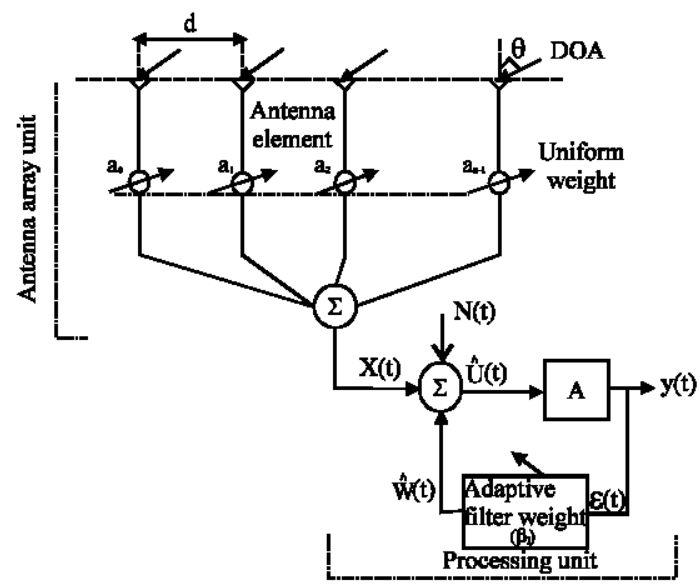

Fig. 2: An adaptive antenna and adaptive filter for S-PCN mobile terminal

elements and there is no significant difference in $\theta$ the Direction-of-Arrival (DOA) of a particular plane wave at any two elements. For $\mathrm{N}$ elements array of identical characteristics, the first pulse is taken as the phase centre, $f(\tau)$.

The second pulse is advanced by $\tau$ and denoted by $f(t-\tau)$, the thirdpulse is advanced by $2 \tau$ and denoted by $f(t-2 \tau)$ and so on. For convenience, five elements are used, so:

$$
\begin{aligned}
f_{T}(t) & =a_{0} f(t)+a_{1} f(t-\tau)+a_{2} f(t-2 \tau) \\
& +a_{3} f(t-3 \tau)+a_{4} f(t-4 \tau)
\end{aligned}
$$

Where $a_{0}, a_{1} \ldots a_{5}$ are the weights/gains of each element of the array. In adaptive antenna array, the weights/gains of each element are taken to be the same (i.e., $\mathrm{a}_{0}=\mathrm{a}_{1}=\mathrm{a}_{4}$ ), conforming to practical situation and the elements to be equally spaced. Had the weights variable, there is possibility they may modulate the desired signal. Now, if the first pulse is Fourier transformed and is represented by $f_{\Delta}(\omega)$, then;

$$
\begin{gathered}
f_{T}(\omega)=a_{0} f_{\Delta}(\omega)+a_{1} f_{\Delta}(\omega) e^{-j \omega \tau}+a_{2} f_{\Delta}(\omega) e^{-j 2 \omega \tau}+ \\
a_{3} f_{\Delta}(\omega) e^{-j 3 \omega \tau}+a_{4} f_{\Delta}(\omega) e^{-j 4 \omega \tau}
\end{gathered}
$$

By multiplying Eq. 2 by $\mathrm{e}^{-j \omega \tau}$ and then subtracting the resulting expression from Eq. 1 and rearranging ensuing expression and using known geometric series expansion we have:

$$
f_{\tau}(\omega)=a_{0} f_{\Delta}(\omega)\left[e^{-j 2 \omega \tau}\left(\frac{\sin (5 \pi f \tau)}{\sin (\pi f \tau)}\right)\right]
$$

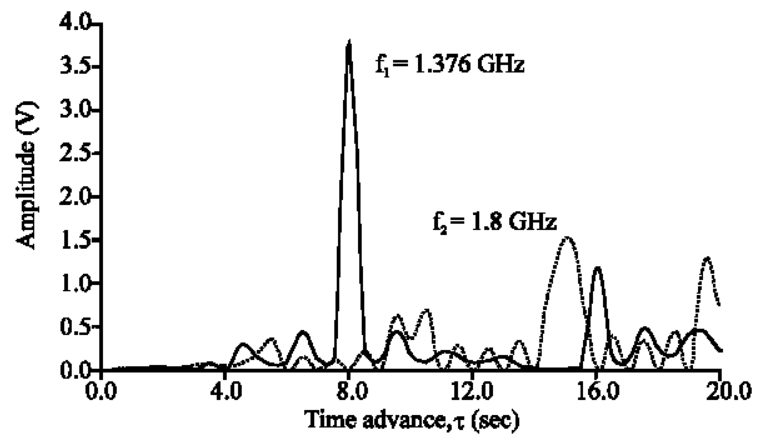

Fig. 3: Antenna array's response at typical S-PCN frequency

Typical S-PCN frequencies of 1.376 and $1.80 \mathrm{GHz}$ (Lutz, 1998; Kolawole, 2009) are used to examine the antenna array's behaviour using Eq. 3. Resulting graphs are shown in Fig. 3. The sidelobes performance has an important impact on interference received from adjacent satellites (and ground based signals operating on same bands) as well as in determining antenna noise temperature. To successfully eliminate or reduce significantly, the effect of external interference, the sidelobes have to be attenuated if not removed. The sidelobes are cancelled or removed in this paper by the adaptive array process.

\section{Processing unit}

Modelling interferences with adaptive filter: The approach models the effect of interferers on the S-PCN systems using interferences as recursive random processes, $\mathrm{N}_{\mathrm{i}}(\mathrm{t})$ with the array antenna. The input to the processor in Fig. 2 is the inverse Fourier $\mathfrak{S}^{-1}\left(\mathrm{f}_{\mathrm{r}}(\omega)\right)$ of the array output which now becomes $\mathrm{X}(\mathrm{t})$ to the summer. The effect of noise from each antenna element is factored in at the output that is for $\mathrm{n}$ elements with equal average Signal-to-Noise (SNR), the average SNR of the array combiner is $10 \log _{10} \mathrm{n}$ which is higher in $\mathrm{dB}$ than the average SNR of any one of the elements (Ingram et al., 2004). Following Fig. 2, the recursive-filtering algorithm can be represented as a linear, discrete-time ( $t$ ) model of the form:

$$
\begin{gathered}
\hat{U}(t)=X(t)+N(t)-\hat{W}(t) \\
Y(t)=A \hat{U}(t)
\end{gathered}
$$

Where:

$A(t)=$ The system parameter

$\mathrm{W}(\mathrm{t})=$ Optimum adaptive but weighted filter

$\mathrm{N}(\mathrm{t})$ = Additive, uncorrelated system interference, assumed white zero-mean Gaussian and stochastic

$\mathrm{Y}(\mathrm{t})=$ Filter (or measurement) output 
The optimum interference $\mathrm{W}(\mathrm{T})$ is defined as:

$$
\hat{W}(t)=\sum_{i=0}^{k} \beta_{i}(t) N_{t h}(t-i)
$$

Where $\mathrm{N}_{\text {th }}$ is the acceptable interference threshold and $\mathrm{K}$ is the filter order. The proceeding coefficient of the filter can be estimated from the present coefficient and other thresholds:

$$
\beta_{i}(t+1)=\beta_{i}(t)+2 \eta X(t) N_{t h}(t-i)
$$

Where $\eta$ is the convergence constant. The adaptive filter adapts the filter coefficients to achieve desired signal ensuring convergence; that is, minimizing error $\varepsilon(\mathrm{t})$ at each time index:

$$
\varepsilon(t)=Y(t)-A \hat{U}(t)
$$

Ensuring fast convergence a local minimum is sought leading to establishing threshold value i.e.,

$$
\xi(t)=\sum_{j=0}^{k}\left|\beta_{t}(j)-\beta_{t-1}(j)\right| \leq \alpha_{t h}
$$

Where $\alpha_{\text {th }}$ local minimum threshold. The adaptation gain $\mathrm{g}(\mathrm{m})$ is introduced for coefficient updating recursion for the period of the signal measurement:

$$
\mathrm{g}(\mathrm{m})=\left|\sum_{\mathrm{i}=0}^{\mathrm{m}} \frac{\hat{\mathrm{U}}(\mathrm{i})}{\mathrm{X}(\mathrm{i})+\mathrm{N}(\mathrm{i})}\right|
$$

Where $\mathrm{m}$ is the period which the mobile terminal engages the network. As shown in Fig. 4, application of adaptive filter in conjunction with the antenna array processing shows encouraging responses in terms of interference reduction. The implication of our method is that the variation of the filter's weights as a result of movement of the mobile users may affect the effectiveness of the system as the users move from orbit to anothe; Low Earth Orbit (LEO) through to Geostationary Orbit (GEO) for example. However, a mobile user of any of the systems might experience the same problem since the environment is changing too rapidly for the weights to converge. This is an area that is being investigated. Also, the relative motion between the user and the satellite reference may be another source of error resulting in Doppler shift. Recent study of Rao et al. (2009) suggests that using frequency lock loop could compensate for this error. Whilst the technique has utilised uniform weight for the antenna array, this should not a problem if variable weights were to be utilised (Iwasokun, 2010).

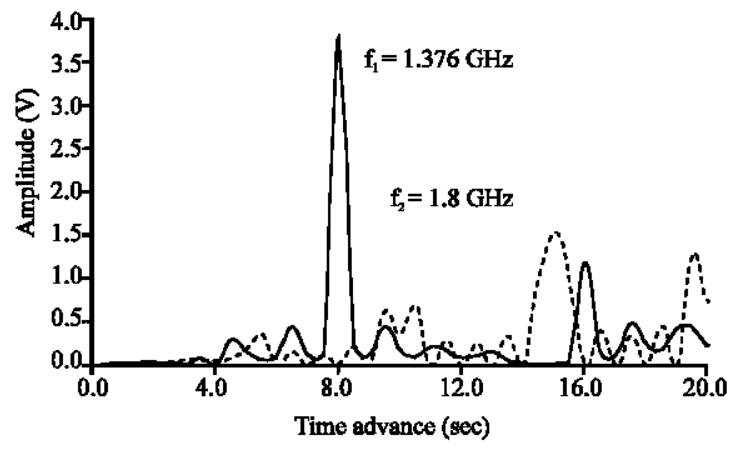

Fig. 4: Simulated recived response of mobile at typical $\mathrm{S}-\mathrm{PCN}$ frequncies

Moreover, since some of the satellite constellations utilise Code Division Multiple Access (CDMA) as the multiple access technique, pseudorandom noise codes can also be utilized to monitor the adaptive array weights.

\section{CONCLUSION}

This study has presented an antenna-array plus adaptive filtering model as a way of eliminating the effect of interference in satellite personal communication systems. This technique has shown that it can eliminate an uncorrelated signals residing in the antenna sidelobes successfully. This technique is easily adaptable to S-PCN in LEO operational environment as a result of shorter time required by LEO satellites to move across the sky.

\section{REFERENCES}

Conte, R., 2005. Satellite rural communications: Telephony and narrowband networks. Int. J. Satell. Commun. Networking, 23: 307-321.

Ghedia, L., 1999. Satellite Personal Communication Networks. Institution of Electrical Engineers, UK.

Ingram, M.A., R. Romanofsky, R.Q. Lee, F. Miranda and Z. Popovic et al., 2004. Optimizing satellite communications with adaptive and phased array antennas. Proceedings of the Earth Science and Technology Conference. http:/www.esto.nasa.gov/ conferences/estc2004/papers/a9p4.pdf.

Iwasokun, S.E., 2010. Elimination of interference and multipath signals in satellite personal communication systems using adaptive antenna array. M. Eng. Thesis, Federal University of Technology, Akure, Nigeria.

Kolawole, M.O., 2003. Radar Systems, Peak Detection and Tracking. Elsevier, Oxford, UK.

Kolawole, M.O., 2009. A Course in Telecommunication Engineering. S. Chand, New Delhi, pp: 240-248. 
Lutz, E., 1998. Issues in satellite personal communication systems. Wireless Networks, 4: 109-124.

Rao, M., L.L. Presti, M. Fantino and G. Garbo, 2009. A software receiver adaptive phase lock loop method. Porceedings of the International Global Navigation Satellite Systems Society (IGNSS) Symposium, Dec. 1-3, Holiday Inn Surfers Paradise, Qld, Australia, pp: 1-13.
Septiawan, R., 2004. Multiservice traffic allocation in LEO satellite communications. Ph.D. Thesis, Bond University, Australia.

Skolnik, M.I., 1981. Introduction to Radar Systems. McGraw-Hill, Singapore. 